\title{
Investigational New Drugs, Volume 25, Number 1
}

\section{Springer Publishing Department}

Published online: 22 March 2007

(C) Springer Science + Business Media, LLC 2007

The publisher apologizes for the error that occurred in this issue of Investigational New Drugs, Vol. 25., No. 1. The incorrect volume year was listed on the first line of each article. It is in fact the 2007 Investigational New Drugs volume.

The online version of the original articles can be found at: http://dx.doi.org/10.1007/s10637-006-7773-9. http://dx.doi.org/10.1007/s10637-006-7589-7. http://dx.doi.org/10.1007/s10637-006-7774-8. http://dx.doi.org/10.1007/s10637-006-9000-0. http://dx.doi.org/10.1007/s10637-006-9008-5. http://dx.doi.org/10.1007/s10637-006-9013-8. http://dx.doi.org/10.1007/s10637-006-8220-7. http://dx.doi.org/10.1007/s10637-006-9006-7. http://dx.doi.org/10.1007/s10637-006-9005-8. http://dx.doi.org/10.1007/s10637-006-9004-9. http://dx.doi.org/10.1007/s10637-006-9003-x.

Springer Publishing Department $(\varangle)$

Norwell, MA 02061, USA 